\title{
Reconfigurer la classe en équipe de recherche à l'ère des TIC
}

Le cas du Québec

Reconfiguring classrooms into research teams in the era of ICT. The case of Quebec

Transformar la clase en equipo de investigación en la era de las TIC. El caso de Quebec

Stéphane Allaire

\section{OpenEdition} Journals

Édition électronique

URL : http://journals.openedition.org/ries/3629

DOI : 10.4000/ries.3629

ISSN : 2261-4265

Éditeur

Centre international d'études pédagogiques

Édition imprimée

Date de publication : 1 décembre 2013

Pagination : 147-153

ISBN : 978-2-85420-601-2

ISSN : $1254-4590$

Référence électronique

Stéphane Allaire, "Reconfigurer la classe en équipe de recherche à l'ère des TIC », Revue internationale d'éducation de Sèvres [En ligne], 64 | décembre 2013, mis en ligne le 01 décembre 2015, consulté le 19 avril 2019. URL : http://journals.openedition.org/ries/3629; DOI : 10.4000/ries.3629 


\section{Reconfigurer la classe en équipe de recherche à l'ère des TIC}

\section{Le cas du Québec}

\section{Stéphane Allaire}

Elle semble bien lointaine, l'époque où Joseph Schwab a défini le concept de situation éducative comme étant quelqu'un qui enseigne quelque chose à quelqu'un dans un contexte donné. Plus spécifiquement, à ce moment, en milieu scolaire, le contexte en question référait essentiellement à la classe physique, ce lieu situé entre quatre murs, porte fermée, dont les activités pouvaient même se dérouler en vase clos de celles du reste de l'école. Aussi, un aspect saillant de la définition de Schwab (1973) est la centration sur l'enseignant, ce qui suggère qu'une situation éducative implique en quelque sorte la principale transfusion d'un contenu d'une personne à une autre.

Depuis le milieu des années 1990, la prolifération de l'Internet dans la sphère personnelle met une pression croissante sur l'école. Les discours souhaitant sa mise à jour, sa reconfiguration, sont abondants. L'école doit se mettre au goût du jour, souhaite-t-on. Elle est déconnectée du "vrai monde ", clame-t-on. Cela est particulièrement prégnant depuis quelques années, avec l'explosion des médias sociaux et l'ubiquité d'accès au réseau mondial à partir des appareils mobiles. En outre, l'évolution de la compréhension de la façon dont l'apprentissage humain s'effectue, notamment par les travaux menés en sciences de l'apprentissage (learning sciences) (Khine \& Saleh, 2010), est un autre élément qui fait pression sur l'école. À cet effet, nombreux sont les systèmes éducatifs, dont celui du Québec qui, depuis le début des années 2000, ont entamé un virage pour, disait-on à l'époque, "passer du paradigme de l'enseignement à celui de l'apprentissage ». Aujourd'hui, ce sont les compétences du XXI ${ }^{e}$ siècle ${ }^{1}$ qui sont au cœur des échanges à propos des orientations pédagogiques.

Progressivement - trop lentement diront certains -, la classe traditionnelle se redéfinit en classe en réseau, c'est-à-dire une classe dont les frontières physiques, bien que toujours présentes, deviennent poreuses, par l'accès à l'Internet. Des jeunes et un enseignant se regroupent toujours dans un lieu physique commun, en l'occurrence une classe située dans une école, mais ils ont la possibilité d'accéder à des ressources auxquelles ils n'avaient pas accès auparavant. La consultation ponctuelle d'informations sur le web, à partir d'un poste informatique unique ou d'un tableau numérique interactif, pour répondre en

1. http://www.p21.org/ 
temps réel ne serait-ce qu'à une question dont la classe n'a pas la réponse, est un premier jalon vers la reconfiguration de la classe traditionnelle (Laferrière, 1999). Elle décentre déjà un peu la responsabilité des contenus du seul enseignant.

Un autre jalon de la classe en réseau, beaucoup plus avancé celui-là, consiste à envisager la classe comme une équipe d'apprentis-chercheurs accompagnés qui investiguent dans un contexte en réseau. Cela contraste avec le concept initial de situation éducative de Schwab. Issus de travaux que nous avons menés (Allaire \& Lusignan, 2011 ; Allaire, Beaudoin, Breuleux, Hamel, Inchauspé, Laferrière \& Turcotte, 2006), nous présentons quelques éléments d'une restructuration de l'espace «classe » qui permet de concilier un usage des TIC avec des principes d'apprentissage contemporains.

\section{Centrer l'activité DE LA CLASSE SUR LA RÉSOLUTION D'UN PROBLÈME COMPLEXE}

Dans une classe organisée classiquement, l'enseignant est souvent prompt à présenter des contenus. L'image qui vient en tête est celle du maître, situé sur l'estrade à l'avant de la salle de classe, exposant son savoir aux élèves qui lui font face et dont les pupitres sont disposés en rang d'oignons. En outre, l'enseignant s'affaire à organiser les contenus logiquement, pour que leur réception par les élèves soit vraisemblablement plus efficace. Dans une classe d'apprentis-chercheurs, c'est le questionnement des élèves qui est d'abord mis en avant. Ils sont amenés à cerner l'état de leurs connaissances de groupe sur une thématique reliée au curriculum. Ils identifient progressivement des zones à éclaircir, qu'ils formuleront en problème à résoudre, en questions à élucider. Tout problème, toute question pour lesquels quelqu'un possède rapidement la réponse, incluant l'enseignant ou les outils de recherche de base de l'Internet, sont reformulés de façon plus complexe, afin qu'une mobilisation collective soit réellement nécessaire. Ainsi, les questions d'ordre descriptif ou énumératif sont généralement évacuées. Une fois l'activité de classe réfléchie en termes de résolution de problème, on peut penser à en ouvrir avantageusement les frontières à l'aide des TIC.

Sur le plan de l'organisation physique, la mobilisation d'un questionnement collectif complexe fécond et porteur pour la classe passe par une disposition qui induit la collaboration. Un premier mouvement peut consister à regrouper les pupitres de deux ou quatre élèves pour amener ces derniers à identifier librement, en sous-groupe, les premières idées qu'ils ont à propos de la thématique ciblée. Dans un second mouvement, les idées sont mises en commun en plénière, où l'enseignant occupe alors un rôle de médiation dans l'identification de points communs et distinctifs entre les sous-groupes, pour parvenir à une organisation collective du travail. Une autre façon consiste à 
procéder en plénière dès le départ, en faisant un grand cercle (ou un U) avec les pupitres, en y incluant celui de l'enseignant. Avec les élèves plus jeunes, lorsque l'espace est suffisant, le cercle peut être formé en s'asseyant directement sur le sol. En outre, il est souhaitable de prévoir un dispositif commun de prise de notes, que la classe peut consulter en temps réel pendant la discussion. Ici, l'usage d'un tableau numérique interactif est tout indiqué. Bien que plus limitatif en termes d'organisation des idées, le tableau conventionnel peut aussi convenir.

\section{SE MÉFIER DE L'IMPRESSION D'INSTANTANÉITÉ D'APPRENTISSAGE}

Les options technologiques sont nombreuses et, bien qu'on dise souvent qu'une technologie n'est qu'un outil, elle est néanmoins susceptible d'induire un usage. C'est ce qu'on appelle "l'effet d'affordance »(Allaire, 2006). L'instantanéité de l'usage personnel de certains médias sociaux gagne à être réfléchie minutieusement dans la perspective d'un usage à des fins d'apprentissage. Un apprentissage en profondeur, c'est-à-dire qui amène les élèves à comprendre et à tisser des liens explicites entre les connaissances d'une discipline donnée, nécessite du temps, en raison du travail de construction et de restructuration cognitive requis. Cela apparaît fort différent des apprentissages spontanés que tout un chacun peut faire chaque jour, lorsqu'il consulte le web à la carte pour répondre à des interrogations factuelles ponctuelles.

Des changements inopinés et fréquents de l'organisation physique, dans un court laps de temps, peuvent induire une perception d'instantanéité. Par exemple, au nom d'un soi-disant besoin de varier les activités, on passe parfois d'un travail individuel à son pupitre, à une réorganisation en îlots de quatre, puis à un agencement plénier en moins d'une heure. Une telle promptitude est à même de questionner le support temporel offert à l'approfondissement.

\section{UTILISER UNE TECHNOLOGIE QUI PERMET DE GARDER DES TRACES}

Puisqu'un apprentissage en profondeur requiert du temps et qu'un questionnement complexe ne peut être élucidé au premier instant, il importe que les apprentis-chercheurs disposent d'un outil leur permettant de travailler de façon itérative. Les résultats de nos travaux préconisent l'usage d'outils technologiques d'écriture asynchrone collective, comme les forums électroniques, puisqu'ils suggèrent plus explicitement la négociation de sens. Par exemple, dans le cadre de deux études portant sur l'apprentissage de l'écriture avec un blogue, peu de traces d'une telle négociation ont pu être documentées (Allaire, Thériault, Gagnon et al., 2013 ; Allaire, Thériault, Gagnon et al., 2011). Qui plus est, il 
importe que ces outils permettent à chaque élève de retravailler ses idées et de les mettre en lien avec celles des autres. Cela contribue à l'élaboration d'une compréhension collective et à un discours de construction de connaissances (Laferrière, Allaire, Breuleux et al., 2009). En restructurant la classe ainsi, il devient possible de s'affranchir des limites de volatilité du discours oral. En ce sens, le dicton «les paroles s'envolent; les écrits restent» demeure porteur d'orientations modernes quant à la façon dont l'apprentissage se réalise.

Quant à une organisation physique de la classe qui supporte un discours de co-construction de connaissances par l'asynchrone, la tentation peut être forte de déplacer momentanément les élèves au laboratoire informatique, pour que chacun dispose d'un ordinateur, ou encore de demander à chaque élève de prendre son portable ou sa tablette, là où de telles initiatives technologiques sont de plus en plus présentes. En ce qui nous concerne, qu'il s'agisse de l'un ou l'autre contexte, il demeure un principe d'organisation du travail dont on gagne à se méfier : celui où " tout le monde fait la même chose au même moment ». Le cas échéant, on risque de retrouver un discours de type monologue collectif, c'est-à-dire un discours de répétition de propos semblables, puisque la classe se concentrera, la plupart du temps, sur l'activité de contribuer plutôt que sur celle de lire les idées dont on a conservé les traces. Dans un contexte de résolution de problème complexe en collectivité, il va de soi que cela est peu souhaitable. En ce sens, une organisation physique de base efficace devrait mettre à la disposition de la classe quatre ou cinq ordinateurs rassemblés dans un, voire deux de ses coins. Là où chaque élève dispose de son propre outil (ordinateur ou tablette), il nous apparaît souhaitable de songer à une organisation multitâche du travail (par exemple selon un plan de travail flexible), afin que tous les élèves ne se retrouvent pas simultanément en train de contribuer à l'outil d'écriture asynchrone.

\section{OSER DOUTER DES INFORMATIONS PROVENANT DE SOURCES DE RÉFÉRENCE}

L'organisation traditionnelle d'une classe consiste à se référer à des sources d'autorité pour s'enquérir de la «vérité ». Une équipe d'apprentischercheurs apprend à prendre ses distances et à chercher à les améliorer. Il s'agit d'un élément particulièrement difficile à mettre en œuvre et, hélas, l'usage de sources de référence a souvent pour effet de mettre un terme à l'entreprise de résolution de problèmes. Une étape intermédiaire consiste à valider la véracité d'une information donnée à partir de plusieurs sources. Par ailleurs, la question de l'usage d'informations pour alimenter le questionnement du groupe dépasse la consultation de documents, qu'ils soient en format papier ou numérique. Par exemple, pour des questionnements en sciences, il est opportun que les élèves élaborent une expérience pour vérifier certaines idées mises en avant, 
certaines hypothèses. Une même expérience peut être reconduite à différentes reprises, en guise de validation, et les résultats rapportés par écrit pour alimenter les idées élaborées par l'équipe.

Ici, l'espace de la classe peut prendre différentes configurations. Pour amener les élèves à discuter de sources d'autorité plutôt qu'à les tenir pour acquises, la structure de collaboration en cercle peut ici aussi être mise à profit. En intégrant le cercle plutôt qu'en adoptant une position frontale, l'enseignant contribue à diminuer la perception de détention du savoir ainsi que de sa validation. En outre, une collecte de données servant à confirmer ou infirmer des hypothèses peut momentanément recadrer l'espace de la classe en l'élargissant à sa communauté immédiate. C'est le cas par exemple d'élèves qui cherchent à comprendre le fonctionnement d'une époque de la société québécoise et qui rendent visite à des personnes âgées dans un centre d'hébergement situé près de l'école, pour s'enquérir de leur vécu, avant de revenir en discuter en classe et d'en conserver des traces sur l'outil d'écriture asynchrone.

\section{ÉTABLIR UN PARTENARIAT EXPLICITE ET COMPLÉMENTAIRE AVEC UNE CLASSE D'UNE AUTRE ÉCOLE}

On transpose parfois le potentiel interactionnel de l'usage personnel des médias sociaux au contexte scolaire, pensant que quiconque, où qu'il soit, alimentera allègrement le discours de la classe au moment qui convient à cette dernière. Nos résultats révèlent que cela relève davantage de l'utopie que de la réalité et qu'il est préférable de structurer un partenariat entre classes au préalable. Lorsque cela se fait, une authenticité accrue en découle habituellement, ce qui est susceptible de contribuer à la motivation des apprentis-chercheurs, mais aussi à une complémentarité supplémentaire en matière de développement des idées. Cela peut aussi raffermir la dynamique de la classe locale : on se prépare ensemble pour rencontrer une autre équipe et les rétroactions obtenues de cette autre équipe permettent d'alimenter l'avancement local, et ainsi de suite. Ces rencontres se déroulent de différentes façons. Ce peut être en plénière alors que la classe distante est projetée au TNI et que les enseignants des classes en présence se chargent de la gestion des droits de parole entre les élèves. Ces rencontres peuvent aussi avoir lieu entre groupes d'élèves à distance. Dans ces cas, quelques ordinateurs sont nécessaires dans chaque classe où des élèves apprennent ensemble. Ces ordinateurs doivent idéalement être placés un peu en retrait des pupitres pour minimiser le bruit ambiant qui peut interférer dans la communication, tout en permettant aux enseignants de garder un oil sur la réalisation du travail effectué. Dans les deux cas, il importe de préciser que les élèves et l'enseignant des classes en présence demeurent physiquement dans leur classe d'appartenance. La visioconférence devient en fait un canal permettant 
d'entrer en contact avec des gens qui seraient, sans lui, impossibles à fréquenter, compte tenu de l'envergure du territoire géographique. On souhaite consolider, voire bonifier la dynamique locale par l'ouverture sur le monde.

Le travail entre classes pose toutefois un enjeu important au niveau de l'organisation scolaire puisque, s'il sous-tend un minimum de coordination entre les classes, en amont de cela, c'est aussi toute la question de la mise en réseau d'équipes d'apprentis-chercheurs intéressées à investiguer avec d'autres qui est présente. Dans l'initiative panquébécoise de l'École en réseau ${ }^{2}$, qui vise à enrichir l'environnement d'apprentissage de petites écoles rurales en faisant apprendre ensemble des classes géographiquement distantes, l'équipe de recherche-intervention assume un rôle important en cette matière, en se tenant au courant des intentions pédagogiques des classes. Plus récemment, Skype in the classroom a été lancé pour offrir un service de "réseautage » entre éducateurs. C'est dire à quel point les frontières de la classe s'estompent, tout en demeurant tracées, ce qui donne naissance à un niveau d'interaction pédagogique supplémentaire.

\section{PRÉVOIR dES MOMENTS D'OBJECTIVATION DES DÉCOUVERTES}

L'objectivation des apprentissages est un concept pédagogique bien connu et concourt à l'intériorisation des nouveaux savoirs acquis. Dans une classe restructurée en réseau, elle possède aussi une fonction qui s'apparente à la publication de travaux scientifiques. Le travail effectué n'est pas seulement envisagé comme source d'un apprentissage individuel mais aussi comme vitrine d'un travail collectif. En ce sens, un site web ou un blogue visant la diffusion d'artéfacts issus de l'investigation collective ${ }^{3}$ devient un complément intéressant à la dynamique interactive de la classe en réseau. La visioconférence peut aussi être mise à contribution, pour organiser l'équivalent d'un colloque.

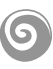

Quelques éléments clés contribuent à restructurer la classe en classe en réseau d'apprentis-chercheurs. Si les technologies de collaboration offrent un potentiel considérable pour tenir compte de principes d'apprentissage contemporains, l'enjeu du déploiement de ces principes ne réside pas tant dans l'usage des outils à proprement parler que dans la façon de procéder à un «design social " grâce à eux, afin de donner lieu à des interactions fécondes. En outre, le design de l'espace devient pluriel et requiert une réflexion, voire une créativité qui vont au-delà du modèle frontal classique.

2. http://www.eer.qc.ca/

3. À titre d'exemple, voir http://eer.qc.ca/petits-chercheurs/ 


\section{BIBLIOGRAPHIE}

ALLAIRE S., THÉRIAULT P., GAGNON V., NORMANDEAU L. \& GAUDREAULT M. (2013) : Étude de cas multiples sur le développement de l'écriture dans des classes du secondaire utilisant le blogue. Rapport de recherche présenté au Ministère de l'Éducation, du Loisir et du Sport. Saguenay: Université du Québec à Chicoutimi. 106 pages. [En ligne]. Disponible : http://constellation.uqac.ca/2450/

ALLAIRE S. \& LUSIGNAN G. (2011) : Enseigner et apprendre en réseau : collaborer entre écoles distantes à l'aide des TIC. Anjou : Éditions CEC.

ALLAIRE S., THÉRIAULT P., GAGNON V. \& LALANCETTE E. (2011) : Environnements d'apprentissage intégrant le blogue au primaire: de la dimension affective à la dimension cognitive de l'écriture. Rapport de recherche présente au Ministère de l'Éducation, du Loisir et du Sport. Saguenay: Université du Québec à Chicoutimi. 90 pages. [En ligne]. Disponible: http://affordance.uqac.ca/publications/RapportBlogues2010- 2011-v19.pdf

ALLAIRE S., BEAUDOIN J., BREULEUX A., HAMEL C., INCHAUSPÉ P., LAFERRIÈRE T. \& TURCOTTE S. (2006) : L'École éloignée en réseau - Rapport final (Phase I). CEFRIO et Ministère de l'Éducation, du Loisir et du Sport. 123 pages. [En ligne]. Disponible : http://eer.qc.ca/doc/2006/12/EERphase2rapportfinal.pdf

ALLAIRE S. (2006) : Les affordances socionumériques d'un environnement d'apprentissage hybride en soutien à des stagiaires en enseignement secondaire. De l'analyse réflexive à la coélaboration de connaissances. Thèse de doctorat, Université Laval.

LAFERRIÈRE T., ALLAIRE S., BREULEUX A., HAMEL C., TURCOTTE S., GAUDREAULT-PERRON J., BEAUDOIN J. \& INCHAUSPÉ P. (2009) : L'École éloignée en réseau: l'apprentissage des élèves. Rapport synthèse 2006-2008 (Phase 3). CEFRIO. 60 pages. [En ligne.] Disponible : http://www.cefrio.qc.ca/fichiers/documents/projets/ eer/ecole_eloignee_reseau_phase3_final_mars09.pdf

KHINE M. \& SALEH I. (2010) : New science of learning: cognition, computers and collaboration in education. London : Springer.

LAFERRIÈRE T. (1999): Apprendre à organiser et à gérer la classe, communauté d'apprentissage assistée par l'ordinateur multimédia en réseau. Revue des sciences de l'éducation, 25(3), 571-591.

SCHWAB J. (1973): The practical 3: translation into curriculum. School Review, 81(4), 501-522. 
